\title{
Injective hyperbolicity of domains
}

\author{
by Marius Overholt (Tromsoe)
}

\begin{abstract}
The pseudometric of Hahn is identical to the Kobayashi-Royden pseudometric on domains of dimension greater than two. Thus injective hyperbolicity coincides with ordinary hyperbolicity in this case.
\end{abstract}

1. Introduction. The Kobayashi pseudodistance $d_{M}$ and KobayashiRoyden pseudodifferential metric $K_{M}$ of a complex manifold $M$ are defined by means of extremal problems for holomorphic mappings of the unit disk $\mathbb{D}$ into $M$. By restricting to injective holomorphic mappings in these extremal problems, one arrives at a pseudodistance $\tau_{M}$ and a pseudodifferential metric $S_{M}$ respectively. These were considered first on plane domains by Siu [4], and in general by Hahn [1]. In the literature, they go under the names of S-metric or Hahn metric. If the pseudodifferential metric $S_{M}$ satisfies an inequality

$$
S_{M}(z, \xi) \geq c\|\xi\|, \quad c>0,
$$

at each point of $M$, then $M$ is said to be S-hyperbolic (alternatively Hahn hyperbolic or injective hyperbolic). In this note we consider $S_{M}$ and its relationship to $K_{M}$.

From the work of Siu [4] and Minda [3] it is known that if $M$ is a Riemann surface, then it is S-hyperbolic unless it is the plane or the extended plane, and Minda also proved that $S_{M}$ and $K_{M}$ are distinct unless $M$ is simply connected. For domains of higher dimension there are results on S-hyperbolicity due to Zhang [7], Vesentini [5] and Vigué [6]. Zhang proved that if $S_{M}$ is a complete metric, then $M$ is a domain of holomorphy, and observed that the converse does not hold. Vesentini showed that a domain of the form $\mathbb{C}^{*} \times \Omega$ is not S-hyperbolic if $\Omega$ is a domain of dimension two or larger, thus disproving the claim by Hahn that $\left(\mathbb{C}^{*}\right)^{n}$ is S-hyperbolic for

1991 Mathematics Subject Classification: Primary 32H15.

Key words and phrases: invariant metric, Kobayashi-Royden metric, Hahn metric, S-metric. 
any positive integer $n$. Vigué generalized the result of Vesentini by showing that a product of two domains is S-hyperbolic only if it is hyperbolic.

The present research was done while the author was on sabbatical at Stanford University, whose hospitality is gratefully acknowledged.

\section{Domains in high dimensions}

Theorem 1. If $\Omega \subseteq \mathbb{C}^{n}, n \geq 3$, is a domain, then $S_{\Omega} \equiv K_{\Omega}$.

Proof. Let $a \in \Omega, \eta \in \mathbb{C}^{n}$ with $\eta \neq 0$ be given. It is enough to show that $S_{\Omega}(a, \eta) \leq K_{\Omega}(a, \eta)$. By a translation of $\Omega$ we may assume that $a=0$, and by a rotation, we may assume that $\eta_{1} \ldots \eta_{n} \neq 0$. Let $\varepsilon>0$ be arbitrary.

Choose a holomorphic mapping $f: \mathbb{D} \rightarrow \Omega$ with $f(0)=0$ and

$$
f_{*}(0) \nu=\eta, \quad|\nu| \leq K_{\Omega}(0, \eta)+\varepsilon / 2
$$

for some $\nu \in \mathbb{C}$. Define $f_{1}: \mathbb{D} \rightarrow \Omega$ by $f_{1}(z)=f((1-\delta) z)$ for a suitably small $\delta>0$; then $f_{1}(0)=0$ and

$$
\left(f_{1}\right)_{*}(0) \frac{\nu}{1-\delta}=\eta, \quad\left|\frac{\nu}{1-\delta}\right| \leq K_{\Omega}(0, \eta)+\varepsilon,
$$

say. Since $f_{1}$ is holomorphic on $\overline{\mathbb{D}}$ and $\operatorname{dist}\left(f_{1}(\overline{\mathbb{D}}), \partial \Omega\right)>0$, there exists a polynomial mapping $f_{2}: \overline{\mathbb{D}} \rightarrow \Omega$ with $f_{2}(0)=0$ and $\left(f_{2}\right)_{*}(0)=\left(f_{1}\right)_{*}(0)$. We write out $f_{2}$ explicitly:

$$
f_{2}(z)=\left(\ldots, \sum_{k=1}^{m} A_{j k} z^{k}, \ldots\right), \quad 1 \leq j \leq n .
$$

We shall show that there exist slight perturbations $\widetilde{A}_{j k}$ of the coefficients $A_{j k}, 1 \leq j \leq n, 2 \leq k \leq n$, such that

$$
f_{3}(z)=\left(\ldots, \sum_{k=1}^{m} \tilde{A}_{j k} z^{k}, \ldots\right), \quad 1 \leq j \leq n,
$$

with $\widetilde{A}_{j 1}=A_{j 1}$, is an injective mapping $f_{3}: \mathbb{D} \rightarrow \Omega$. Since $f_{3}(0)=0$ and

$$
\left(f_{3}\right)_{*}(0)=\left(\ldots, \widetilde{A}_{j 1}, \ldots\right)=\left(\ldots, A_{j 1}, \ldots\right)=\left(f_{2}\right)_{*}(0)=\left(f_{1}\right)_{*}(0),
$$

the mapping $f_{3}$ is a competitor in the extremal problem that defines $S_{\Omega}(0, \eta)$, so

$$
S_{\Omega}(0, \eta) \leq\left|\frac{\nu}{1-\delta}\right| \leq K_{\Omega}(0, \eta)+\varepsilon .
$$

Letting $\varepsilon \rightarrow 0, S_{\Omega} \leq K_{\Omega}$ follows. 
It remains to establish that it is possible to choose $f_{3}$ as required. Assume $f_{3}(z)=f_{3}(w)$ for some $z, w \in \mathbb{C}$ with $z \neq w$, thus

$$
\begin{aligned}
& \widetilde{A}_{11} z+\ldots+\widetilde{A}_{1 m} z^{m}=\widetilde{A}_{11} w+\ldots+\widetilde{A}_{1 m} w^{m} \\
& \ldots \ldots \ldots \ldots \ldots \ldots \ldots \ldots \ldots \ldots \ldots \ldots \\
& \widetilde{A}_{n 1} z+\ldots+\widetilde{A}_{n m} z^{m}=\widetilde{A}_{n 1} w+\ldots+\widetilde{A}_{n m} w^{m} .
\end{aligned}
$$

Rearranging and dividing by $z-w$, we obtain

$$
\begin{aligned}
& \widetilde{A}_{12}(z+w)+\widetilde{A}_{13}\left(z^{2}+z w+w^{2}\right)+\ldots=-\widetilde{A}_{11}, \\
& \widetilde{A}_{n 2}(z+w)+\widetilde{A}_{n 3}\left(z^{2}+z w+w^{2}\right)+\ldots=-\widetilde{A}_{n 1} .
\end{aligned}
$$

The image of $\mathbb{C}^{2}$ under the mapping given by

$$
\begin{aligned}
X_{1} & =z+w \\
X_{2} & =z^{2}+z w+w^{2} \\
\ldots \ldots & \cdots \cdots \cdots+\ldots \ldots+\cdots \\
X_{m-1} & =z^{m-1}+\ldots+w^{m-1}
\end{aligned}
$$

lies on a projective surface $V$, while the equations

$$
\begin{gathered}
B_{12} X_{1}+\ldots+B_{1 m} X_{m-1}=1, \\
\ldots \ldots \ldots \ldots \ldots \ldots \ldots \cdots \cdots \cdots \\
B_{n 2} X_{1}+\ldots+B_{n m} X_{m-1}=1,
\end{gathered}
$$

where $B_{j k}=\widetilde{A}_{j k} /\left(-\widetilde{A}_{j 1}\right)$, define a linear subspace $L$ of the projective space $P_{m-1}(\mathbb{C})$ which is generically of dimension $m-1-n$. Thus $V \cap L=\emptyset$ generically $\operatorname{since} \operatorname{dim}(V)+\operatorname{dim}(L)=2+m-1-n=(m-1)-(n-2)<m-1$ when $n \geq 3$. In particular, the set of $B_{j k}$ for which $V \cap L=\emptyset$ is dense in $\mathbb{C}^{n(m-1)}$, and so the set of $\widetilde{A}_{j k}$ for which $f_{3}$ is injective on $\mathbb{C}$ is dense in $\mathbb{C}^{n(m-1)}$. Since $\operatorname{dist}\left(f_{2}(\overline{\mathbb{D}}), \partial \Omega\right)>0$, we can choose the $\widetilde{A}_{j k}$ close enough to the $A_{j k}$ so that $\operatorname{dist}\left(f_{3}(\overline{\mathbb{D}}), \partial \Omega\right)>0$ while keeping $f_{3}$ injective.

This theorem has some of the results of Zhang, Vesentini and Vigué as corollaries in dimension greater than two. From [2] it is known that a domain which is complete hyperbolic is a domain of holomorphy, thus the theorem of Zhang follows for domains of dimension greater than two. Theorem III of [5] follows directly, as does Corollaire 3.2 of [6] in dimension greater than two.

\section{References}

[1] K. T. Hahn, Some remarks on a new pseudo-differential metric, Ann. Polon. Math. 39 (1981), 71-81.

[2] S. Kobayashi, Hyperbolic Manifolds and Holomorphic Mappings, Marcel Dekker, New York, 1970, p. 77. 
[3] C. D. Minda, The Hahn metric on Riemann surfaces, Kodai Math. J. 6 (1983), $57-69$.

[4] Y.-T. Siu, All plane domains are Banach-Stein, Manuscripta Math. 14 (1974), 101105.

[5] E. Vesentini, Injective hyperbolicity, Ricerche Mat., Suppl. Vol. 36 (1987), 99-109.

[6] J.-P. Vigué, Une remarque sur l'hyperbolicité injective, Atti Accad. Naz. Lincei Rend. Cl. Sci. Fis. Mat. Natur. (8) 83 (1989), 57-61.

[7] J. Zhang, Metric S on holomorphy domain, Kexue Tongbao 33 (5) (1988), 353-356.

IMR UNIVERSITY OF TROMSOE

N-9037 TROMSOE, NORWAY

E-mail: MARIUS@MATH.UIT.NO

Reçu par la Rédaction le 29.12.1994 